\title{
SILAC-based phosphoproteomics reveals an inhibitory role of KSR1 in p53 transcriptional activity via modulation of DBC1
}

\author{
H Zhang ${ }^{1}$, Y Xu ${ }^{1}$, A Filipovic ${ }^{1}, \mathrm{~L}$ C Lit ${ }^{1,2}, \mathrm{C}-\mathrm{Y}$ Koo ${ }^{1}$, J Stebbing ${ }^{1}$ and G Giamas ${ }^{*}, 1$ \\ ${ }^{1}$ Division of Cancer, Department of Surgery and Cancer, Imperial College London, Hammersmith Hospital Campus, Du Cane \\ Road, London, W12 ONN, UK and 2Department of Physiology, Faculty of Medicine, University of Malaya, Kuala Lumpur 50603, \\ Malaysia
}

Background: We have previously identified kinase suppressor of ras-1 (KSR1) as a potential regulatory gene in breast cancer. KSR1, originally described as a novel protein kinase, has a role in activation of mitogen-activated protein kinases. Emerging evidence has shown that KSR1 may have dual functions as an active kinase as well as a scaffold facilitating multiprotein complex assembly. Although efforts have been made to study the role of KSR1 in certain tumour types, its involvement in breast cancer remains unknown.

Methods: A quantitative mass spectrometry analysis using stable isotope labelling of amino acids in cell culture (SILAC) was implemented to identify KSR1-regulated phosphoproteins in breast cancer. In vitro luciferase assays, co-immunoprecipitation as well as western blotting experiments were performed to further study the function of KSR1 in breast cancer.

Results: Of significance, proteomic analysis reveals that KSR1 overexpression decreases deleted in breast cancer-1 (DBC1) phosphorylation. Furthermore, we show that KSR1 decreases the transcriptional activity of p53 by reducing the phosphorylation of DBC1, which leads to a reduced interaction of DBC1 with sirtuin-1 (SIRT1); this in turn enables SIRT1 to deacetylate p53.

Conclusion: Our findings integrate KSR1 into a network involving DBC1 and SIRT1, which results in the regulation of p53 acetylation and its transcriptional activity.

Kinase suppressor of ras-1 (KSR1), initially described more than 15 years ago as a novel protein kinase in the Ras-Raf pathway (Kornfeld et al, 1995; Therrien et al, 1995) is an essential scaffolding protein that co-ordinates the assembly of the mitogen-activated protein kinase (MAPK) complex, consisting of the MAPK kinase MEK and the extracellular signal-regulated kinase (ERK) to facilitate activation of MEK and thus ERK (Kolesnick and Xing, 2004; Zafrullah et al, 2009; Goettel et al, 2011). KSR1 has been extensively referred to as a pseudokinase, because of its lack of a key catalytic residue (Zhang et al, 2012). However, emerging evidence suggests that KSR1 functions as an active kinase as well as a scaffold protein. Using recombinant wildtype KSR1, different groups have shown that KSR1 is capable of directly phosphorylating Raf-1 and MEK1 (Zafrullah et al, 2009; Goettel et al, 2011; Hu et al, 2011). On the other hand, crystal structure analysis suggests a direct interaction between KSR and Raf that enables KSR1 to regulate Raf activation independent of its catalytic activity (Rajakulendran et al, 2009). Such findings add complexity to the simple view of ERK spatio-temporal pathway control and identify pseudokinases such as KSR1 as potential targets.

Given the role of KSR1 in the Ras-Raf-MAPKs cascade, intensive efforts have focused on Ras-dependent cancers. For instance, recent studies reported that KSR1 regulates the proliferative and oncogenic potential of cells and inhibition of KSR1 abrogates Ras-dependent pancreatic cancer growth (Xing et al, 2003; Kortum and Lewis, 2004). Similarly, KSR1 is required 
for tumour formation in a skin cancer mouse model (Lozano et al, 2003). As Ras mutations are rare in breast cancer, the involvement of KSR1 may not depend on the canonical Ras-Raf-MAPKs pathway. Using a short interfering RNA (siRNA) kinome screen, we identified KSR1 as a novel regulator of the transcriptional activity of oestrogen receptor alpha (ER $\alpha$; Giamas et al, 2011). Moreover, we show that KSR1 expression is significantly correlated with overall and diseasefree survival in patients with breast cancer (our unpublished data). However, its biologic functions in this setting have remained unexplored, as are its major partner proteins and pathways including those connected to p53, which we implicate here.

The p53 tumour suppressor is well known to have a central role in cell growth arrest, apoptosis and cellular response to genotoxic stress (Levine, 1997; Vogelstein et al, 2000). Its transcriptional activity is highly regulated by post-transcriptional modifications including acetylation (Brooks and $\mathrm{Gu}, 2003$; Tang et al, 2008). Previous data demonstrate that deleted in breast cancer 1 (DBC1) directly interacts and negatively regulates the deacetylase SIRT1 resulting in an increase of p53 acetylation (Kim et al, 2008; Zhao et al, 2008). Furthermore, phosphorylation of DBC1 is necessary for its interaction with SIRT1, while it inhibits the activity of SIRT1 in response to DNA damage (Yuan et al, 2012; Zannini et al, 2012).

Herein, we present a KSR1-regulated phosphoproteomic profile in breast cancer cells using a stable isotope labelling of amino acids in cell culture (SILAC) approach. Furthermore, we identify a role of KSR1 in the regulation of p53 transactional activity by reducing its acetylation via the modulation of DBC1-SIRT1 interaction.

\section{MATERIALS AND METHODS}

SILAC cell culture. SILAC dialysed calf serum and custom Dulbecco's modified Eagle's medium (DMEM) mediums containing either unlabelled $\left[{ }^{12} \mathrm{C}_{6},{ }^{14} \mathrm{~N}_{4}\right]$-arginine (Arg) and $\left[{ }^{12} \mathrm{C}_{6}\right]$-lysine (Lys) (R0K0 -'light') or labelled $\left[{ }^{13} \mathrm{C}_{6},{ }^{15} \mathrm{~N}_{4}\right]$-Arg and $\left[{ }^{13} \mathrm{C}_{6}\right]$-Lys (R10K8 -'heavy') were purchased from Dundee Cell Products (Dundee, UK). MCF7 cells were grown in these custom DMEM mediums along with $10 \%$ dialysed fetal calf serum (FCS) and $1 \%$ of antibiotics (penicillin and streptomycin). The cells that were grown for at least seven passages were used for this experiment.

Protein digestion and peptide fractionation. Equal amounts of protein from unlabelled and labelled samples were combined before protein digestion. Briefly, samples were reduced in $10 \mathrm{~mm}$ dithiothreitol (DTT) and alkylated in $50 \mathrm{~mm}$ iodoacetamide before boiling in loading buffer, and then separated by one-dimensional sodium dodecyl sulphate (SDS)-PAGE 4-12\% Bis-Tris Novex mini-gel from Invitrogen (Paisley, UK) and visualised by colloidal Coomassie staining from Invitrogen. The entire protein gel lanes were excised and cut into 10 slices each. Every gel slice was subjected to in-gel digestion with trypsin overnight at $37^{\circ} \mathrm{C}$. The resulting tryptic peptides were extracted by formic acid (1\%) and acetonitile, lyophilised in a speedvac and resuspended in 1\% formic acid.

Protein digestion and phosphopeptide enrichment. FASP procedure was performed as previously described (Wisniewski et al, 2009). In all, 500 ul FASP 1 (8 $\mathrm{m}$ urea, $20 \mathrm{~mm}$ DDT in $100 \mathrm{~mm}$ Tris/HCL $\mathrm{pH} 8.5$ ) was added to $\sim 2 \mathrm{mg}$ protein lysate to dilute SDS concentration and transferred to a Vivacon 500, 30k MWCO HY filter from Sartorius Stedim Biotech (Epsom, UK). The sample was buffer exchanged using FASP 1 several times by spinning the tube at $7000 \mathrm{~g}$ to remove detergents. The protein lysate was concentrated by centrifugation and diluted in FASP $2(100 \mathrm{~mm}$ Tris/ $\mathrm{HCl}$ $\mathrm{pH}$ 8.5) ready for trypsin digestion. The sample was reduced using $50 \mathrm{~mm}$ fresh IAA in FASP 2 in the dark for $30 \mathrm{~min}$. Lysates were spun down to remove excess IAA and buffer exchanged into FASP 3 (100 mM triethyl ammonium bicarbonate). Trypsin was dissolved in FASP 3 to give a 1:200 enzyme to protein ratio and added in a volume of at least $100 \mu \mathrm{l}$ for $4-6 \mathrm{~h}$. This was repeated with fresh trypsin for a further overnight incubation. Lysates were spun down and washed with $0.5 \mathrm{M} \mathrm{NaCl}$ and $150 \mu \mathrm{l} 10 \%$ TFA added to reduce the $\mathrm{pH}$. A standard desalting procedure was used (Thingholm et al, 2006).

Hydrophilic interaction chromatography (HILIC) fractionation: A TSKgel Amide-80 separation column with a TSKgel Amide guard column was used for HILIC separation of FASP peptides. Buffer A: $80 \%$ ACN, $0.1 \%$ formic acid and buffer B: $0.1 \%$ formic acid was used for the gradient at a flow rate of $0.6 \mathrm{~mJ} \mathrm{~min}{ }^{-1}$. The sample was separated into 45 fractions, collected 2 mins per vial and dried using a speed vac.

$\mathrm{TiO}_{2}$ enrichment was performed as previously described (Thingholm et al, 2006). $\mathrm{TiO}_{2}$ beads were washed and re-suspended in buffer $\mathrm{B}$ at $50 \mu \mathrm{g} \mu \mathrm{l}^{-1}$ and added to tubes to give $1 \mathrm{mg}$ per tube. The sample was re-suspended in loading buffer, added to beads and incubated at RT for $20 \mathrm{~min}$. After washing, using buffers $\mathrm{A}$ and $\mathrm{B}$ samples were eluted using aliquots of $0.5 \%$ $\mathrm{NH}_{4} \mathrm{OH}$. Elutions were pooled and $10 \mu \mathrm{l}$ of $20 \% \mathrm{FA}$ added to adjust the $\mathrm{pH}$. Samples were dried and re-suspended in 1\% FA.

Mass spectrometry methods. Trypsin-digested peptides were separated using an Ultimate 3000 RSLC nanoflow LC system from Thermo Scientific (Cramlington, UK). On average $0.5 \mu \mathrm{g}$ was loaded with a constant flow of $5 \mu \mathrm{min}^{-1}$ onto an Acclaim PepMap100 nanoViper C18 trap column $(100 \mu \mathrm{m}$ inner-diameter, $2 \mathrm{~cm}$; Thermo Scientific). After trap enrichment, peptides were eluted onto an Acclaim PepMap RSLC nanoViper, C18 column (75 $\mu \mathrm{m}, 15 \mathrm{~cm}$; Thermo Scientific) with a linear gradient of $2-40 \%$ solvent B (80\% acetonitrile with $0.08 \%$ formic acid) over $65 \mathrm{~min}$ with a constant flow of $300 \mathrm{nl} \mathrm{min}{ }^{-1}$. The HPLC system was coupled to a linear ion trap Orbitrap hybrid mass spectrometer (LTQ-Orbitrap Velos, Thermo Scientific) via a nano electrospray ion source (Thermo Scientific). The spray voltage was set to $1.2 \mathrm{kV}$, and the temperature of the heated capillary was set to $250^{\circ} \mathrm{C}$. Fullscan MS survey spectra $(\mathrm{m} / \mathrm{z} 335-1800)$ in profile mode were acquired in the Orbitrap with a resolution of 60000 after accumulation of 1000000 ions. The 15 most intense peptide ions from the preview scan in the Orbitrap were fragmented by collision-induced dissociation (normalised collision energy, 35\%; activation Q, 0.250; and activation time, $10 \mathrm{~ms}$ ) in the LTQ after the accumulation of 10000 ions. Maximal filling times were $1000 \mathrm{~ms}$ for the full scans and $150 \mathrm{~ms}$ for the MS/MS scans. Precursor ion charge state screening was enabled, and all unassigned charge states as well as singly charged species were rejected. The lock mass option was enabled for survey scans to improve mass accuracy. Data were acquired using the Xcalibur software from Thermo Scientific.

Quantification and bioinformatics analysis. The raw mass spectrometric data files obtained for each experiment were collated into a single quantitated data set using MaxQuant (Cox and Mann, 2008) and the Andromeda search engine software (Cox et al, 2011). Enzyme specificity was set to that of trypsin, allowing for cleavage $\mathrm{N}$-terminal to proline residues and between aspartic acid and proline residues. Other parameters used were: (i) variable modifications, methionine oxidation, protein $N$-acetylation, gln $\rightarrow$ pyro-glu; (ii) fixed modifications, cysteine carbamidomethylation; (iii) database: target-decoy human MaxQuant (ipi.HUMAN.v3.68); (iv) heavy labels: R6K4 and R10K8; (v) MS/MS tolerance: FTMS - 10 p.p.m., ITMS - 0.6 Da; (vi) maximum peptide length, 6; (vii) maximum missed cleavages, 2; (viii) maximum of labelled amino acids, 3 ; and (ix) false discovery rate, $1 \%$. Peptide ratios were calculated for each arginine-containing and/or lysine-containing peptide as the peak area of labelled arginine/lysine divided by the peak area of non-labelled arginine/ lysine for each single-scan mass spectrum. Peptide ratios for all 
arginine-containing and lysine-containing peptides sequenced for each protein were averaged. Data are normalised using $1 /$ median ratio value for each identified protein group per labelled sample.

Cell lines, reagents, antibodies and plasmids. MCF7, ZR75-1, SKBR3, MDA-MB-231 (MDA231) and p53 ${ }^{+/+}$HCT116 cells were maintained in DMEM supplemented with $10 \%$ FCS and $1 \%$ penicillin/ streptomycin/glutamine. All cells were incubated at $37^{\circ} \mathrm{C}$ in humidified $5 \% \mathrm{CO}_{2}$. FuGENE HD transfection reagent was obtained from Roche (Burgess Hill, UK). siKSR1 were purchased from Qiagen (Crawley, UK) and verified. Plasmids containing human wild-type KSR1 (pCMV6-KSR1) and empty vector (pCMV6-vector) were obtained from OriGene (Cambridge, UK). Mutant KSR1 R502M was generated using QuikChange Site-Directed Mutagenesis Kit were from Stratagene (Stockport, UK) and confirmed by plasmid sequence. MCF7-parental and MCF7-KSR1 stable cells were generated by transfection of either pCMV6-vector or pCMV6-KSR1 in MCF7 cells and selected in the presence of G418 $\left(1 \mathrm{mg} \mathrm{ml}^{-1}\right)$. KSR1 overexpression was confirmed by RT-qPCR and western blotting. Etoposide was purchased from Sigma Aldrich (Gillingham, UK). The Dual-Glo Luciferase Assay System was purchased from Promega (Southampton, UK). The following antibodies were used: KSR1 rabbit polyclonal from Cell Signaling (Hitchin, UK), anti-Flag mouse monoclonal (Sigma Aldrich), p53 mouse monoclonal DO-1 from Santa Cruz (Wiltshire, UK), acetylated-p53 and phospho-p53 Ser15 rabbit polyclonal (Cell Signaling), SIRT1 rabbit polyclonal (Santa Cruz), DBC1 and phosphoDBC1 Thr454 rabbit polyclonal (Cell Signaling) and $\beta$-actin mouse monoclonal from Abcam (Cambridge, UK). The following p53-target gene promoter-reporter constructs previously described (Vikhanskaya et al, 2007) were a kind gift from Professor Kanaga Sabapathy: AIP-1luciferase (luc), IGFBP3-luc, R2-luc and cyclinG1-luc. The renilla luciferase reporter vector ( $\mathrm{pRL}-\mathrm{TK}$ ) was purchased from Promega.

Protein extraction and western blotting. NP40 lysis buffer (50 mm Tris-HCl, $\mathrm{pH} 8.0,150 \mathrm{~mm} \mathrm{NaCl}, 10 \%$ (v/v) glycerol, $1 \%$ NP40, 5 mм DTT, 1 mм EDTA, 1 mм EGTA, $50 \mu \mathrm{m}$ leupeptin and $30 \mu \mathrm{g} \mathrm{ml}^{-1}$ aprotinin) was used to extract whole-cell lysates. Cell pellets were mixed thoroughly with NP40 lysis buffer, and then incubated in ice for $15 \mathrm{~min}$ before centrifuging at 15000 r.p.m. for $15 \mathrm{~min}$ at $4{ }^{\circ} \mathrm{C}$. Nuclear and cytoplasmic extracts were prepared from whole-cell lysis using NE-PER Nuclear and Cytoplasmic Extraction Kit (Thermo Scientific). Protein concentration was measured by the bicinchoninic acid (BCA) protein assay from Pierce (Epsom, UK). Lysates were heated with $5 \times$ SDS sample buffer at $95^{\circ} \mathrm{C}$ for $5 \mathrm{~min}$ before they were loaded to $10 \%$ SDSPAGE. Samples were then transferred to Hybond ECL super nitrocellulose membranes from GE Healthcare (Little Chalfont, UK). Subsequently, the membranes were blocked in TBS containing $0.1 \%(\mathrm{v} / \mathrm{v})$ Tween 20 and $5 \%(\mathrm{w} / \mathrm{v})$ non-fat milk for $1 \mathrm{~h}$. The primary antibodies were probed with membranes overnight at $4{ }^{\circ} \mathrm{C}$. The membranes were then washed three times in TBS/Tween for 15 min following incubation with HRP-conjugated secondary antibodies (1:3000 dilution) for $60 \mathrm{~min}$. The membranes were then washed three times again and were detected with enhanced chemiluminescence (ECL). Films were developed using a Konica SRX-1001A X-ray developer (Banbury, UK). Alternatively, membranes were incubated with IRDye donkey anti-mouse or donkey anti-rabbit secondary antibodies for $60 \mathrm{~min}$ and visualised by Odyssey Fc Imaging System from LI-COR (Cambridge, UK).

RNA extraction and RT-qPCR. RNeasy kit (Qiagen) was used to isolate total RNA. Reverse transcription was performed using highcapacity cDNA reverse transcription kit from Applied Biosystems (Warrington, UK). RT-qPCR analysis was performed on a 7900HT Thermocycler (Applied Biosystems) using TaqMan mastermix and primers for TP53 and GAPDH cDNAs, purchased from Applied Biosystems.
Luciferase reporter assay. In all, $8 \times 10^{4}$ per well MCF7 cells were seeded into 24 -well plate and transfection was performed using FuGENE HD transfection reagent according to the manufacturer's instructions. Cells were transfected with different p53 constructs, and pCMV6-KSR1 or pCMV6-vector and together with renilla luciferase reporter vector (pRL-TK). Cell lysates were collected after 24-h transfection and firefly and renilla luciferase activities were measured by the Dual-Glo Luciferase Assay kit as per the manufacturer's protocols described. The transcriptional activity of various p53 constructs were determined by firefly luciferase activity and normalised against renilla luciferase activity, which was served as control for transfection efficiency.

Immunofluorescence staining. MCF7 cells seeded on glass coverslips in $6 \mathrm{~cm}$ dishes were transfected with pCMV6-KSR1 or pCMV6-Vector using FuGENE HD. Cells were fixed in $4 \% \mathrm{w} / \mathrm{v}$ paraformaldehyde at $37^{\circ} \mathrm{C}$ for $15 \mathrm{~min}$, permeabilised with $0.1 \%$ Triton-X for $10 \mathrm{~min}$ and incubated in immune-fluorescent blocking buffer (10\% AB-serum in PBS) for $1 \mathrm{~h}$, followed by incubation with p53 antibody (DO-1). After washing with PBS, coverslips were incubated for $45 \mathrm{~min}$ at $37^{\circ} \mathrm{C}$ with anti-mouse-IgG Alexa Fluor - 555 antibody (Invitrogen). DNA was visualised by DAPI staining. Cells were examined on an Axiovert-200 laser scanning inverted microscope from Zeiss (Welwyn Garden City, UK) as previously described (Giamas et al, 2011).

Cell proliferation assay. Sulphorhodamine B (SRB) assay was performed to determine the growth of breast cancer cell lines in 96-well plates. After siRNA knockdown of KSR1 at indicated time points, plates were collected for the following protocol. Cells were fixed by adding $100 \mu \mathrm{l}$ per well of ice-cold $40 \%$ trichloroacetic acid (TCA) to each culture for $1 \mathrm{~h}$ and incubated in the fridge. Plates were then washed $\times 5$ times in running tap water (allow the water to fill wells indirectly). Cells were stained with $100 \mu \mathrm{l}$ of $0.4 \%$ (w/v) SRB from Sigma Aldrich in $1 \%$ acetic acid for $30 \mathrm{~min}$ and plates were washed five times in $1 \%$ acetic acid and left to air dry overnight. On the day of plate reading, bound dye was solubilised by adding $100 \mu \mathrm{l}$ of $10 \mathrm{~mm}$ Tris base to all the wells and plates were measured at $492 \mathrm{~nm}$ using Tecan microplate reader (Reading, UK).

Neddylation assay. MCF7 cells plated on $15 \mathrm{~cm}$ dishes were transfected using FuGENE HD with $8 \mu \mathrm{g}$ pcDNA3-HA-NEDD8 from Addgene (Cambridge, MA, USA), $8 \mu \mathrm{g}$ pCMV6-KSR1 or $8 \mu \mathrm{g}$ pCMV6-vector constructs as indicated. After $24 \mathrm{~h}$, cells were lysed in radio-immunoprecipitation assay (RIPA) buffer (Sigma Aldrich) supplemented with protease inhibitors. Total protein was quantified by the BCA assay (Pierce). In all, $2 \mu \mathrm{g}$ mouse IgG or p53 (DO1) was pre-incubated with protein agarose beads for $2 \mathrm{~h}$ to form IP matrix complex (ImmunoCruz IP/WB Optima C System, Santa Cruz). In total, $2 \mathrm{mg}$ protein lysate was added into the beads and was incubated on a rotator overnight at $4{ }^{\circ} \mathrm{C}$. Beads were washed with RIPA buffer for three times and were heated in SDS loading buffer. Neddylated p53 were detected by western blot using p53specific DO-1 antibody or NEDD8 antibody (Cell Signaling) as described before (Xirodimas et al, 2004; Abida et al, 2007).

Statistical analysis. Each experiment was repeated at least three times. Student's $t$-test was two-sided at a 0.05 significance level and performed using SPSS 16.0 statistical software (SPSS Inc., Chicago, IL, USA).

\section{RESULTS}

Phosphoproteomics analysis of KSR1-regulated proteins using SILAC. In order to obtain the KSR1-regulated phosphoproteome profile, a necessary step to understand its integrated signalling pathways in breast cancer, we first performed a quantitative proteomic analysis. 
Here, MCF7 cells were maintained for seven cell divisions in either R0K0 'light' medium, containing unlabelled $\left[{ }^{12} \mathrm{C}_{6},{ }^{14} \mathrm{~N}_{4}\right]$ arginine (Arg) and $\left[{ }^{12} \mathrm{C}_{6}\right]$-lysine (Lys) amino acids, or in $\mathrm{R} 10 \mathrm{~K} 8$ 'heavy' medium, containing labelled $\left[{ }^{13} \mathrm{C}_{6},{ }^{15} \mathrm{~N}_{4}\right]$-Arg and $\left[{ }^{13} \mathrm{C}_{6}\right.$, ${ }^{15} \mathrm{~N}_{2}$ ]-Lys. Labelled cells were then transfected with either the pCMV6 vector (R0K0, control) or with a pCMV6-KSR1 plasmid (R10K8) that encodes for the full-length KSR1. After $24 \mathrm{~h}$, proteins were extracted, mixed 1:1, separated on SDS-PAGE, trypsindigested, fractionated and analysed by LC-MS/MS using MaxQuant software (Cox and Mann 2008; Figure 1A).

We identified a total of 2504 proteins out of which 2032 were quantified (false discovery rate $<1 \%$ ). Similarly, we found 1409 phosphopeptides from 891 phosphoproteins out of which 1165 phosphopeptides from 812 phosphoproteins were quantified. After normalisation, we determined the phosphorylation $v$ s total protein level ratio between control and KSR1-overexpressed samples. Based on our data, we obtained information about the phosphorylation change of 379 potential sites that correspond to 240 proteins, as several proteins had more than one potential phosphorylation sites. Among these modulated sites, 341 phosphoserine (pS), 37 phosphothreonine (pT) and 1 phosphotyrosine (pY) sites were included (Supplementary Excel File 1).

Surprisingly, only 3 out of the 379 identified phospho-sites were induced $>50 \%$ while most of them (233 out of 379 ) were actually decreased $(<50 \%)$ after KSR1 overexpression. These data partly support the characterisation of KSR1 as a pseudokinase, emphasising its primary role as a scaffold protein not a kinase. The values from total and phosphorylated proteins were plotted to create a graph showing the $\log _{2}$ normalised 'total proteins' $v s$ the $\log _{2}$ 'phosphorylated proteins' ratios (Figure 1B).

Ontology analysis of differentially regulated KSR1 phosphoproteins. We then performed gene ontology (GO) analysis and classification (using the 'Panther' software; Mi et al, 2013) to assign the KSR1-regulated phosphoproteome according to (i) molecular functions, (ii) biological processes and (iii) cellular components (Figure 1C). We found significant enrichment for GO molecular functions terms related to binding (GO: 0005488), catalytic activity (GO: 0003824), structural molecule activity (GO: 0005198), as well as transcription regulator (GO: 0003824) and enzyme regulator (GO: 0030234) activities. Our phosphoproteomics analysis facilitated the identification of biological processes associated with cell cycle (GO: 0007049) and communication (GO: 0007154) along with metabolic (GO: 0008152), cellular (GO: 009987) and transport (GO: 0006810) processes. Finally, regarding the localisation of the identified KSR1-regulated phosphoproteins, there was a similar distribution between membrane/cytoplasmic and nuclear cell components, resulting from the direct and indirect effects of

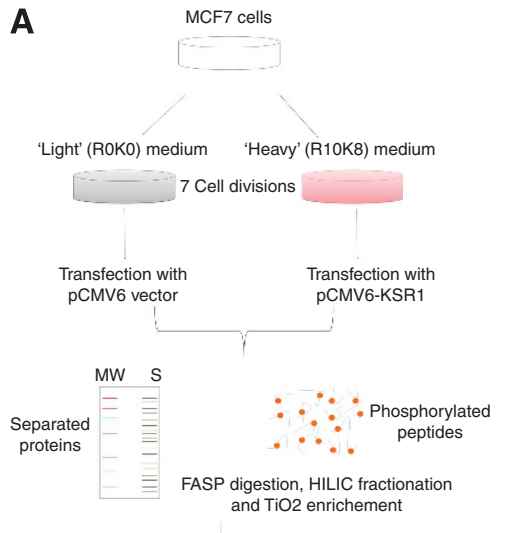

LC - mass spectometry

Protein identification and quantification (MaxQnant)

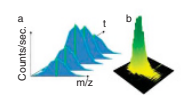

C

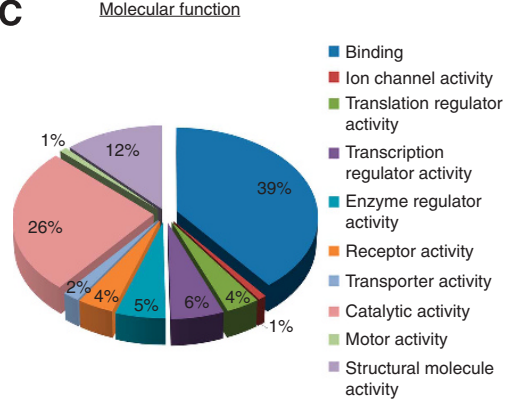

B

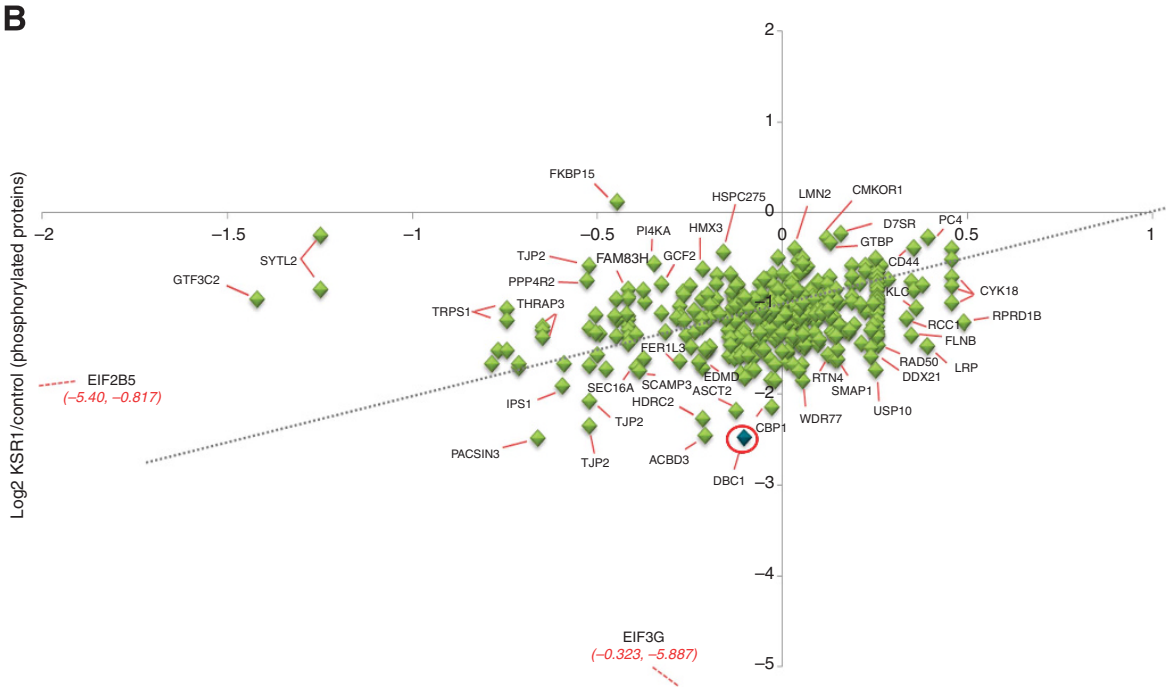

Log2 KSR1/control (total proteins)

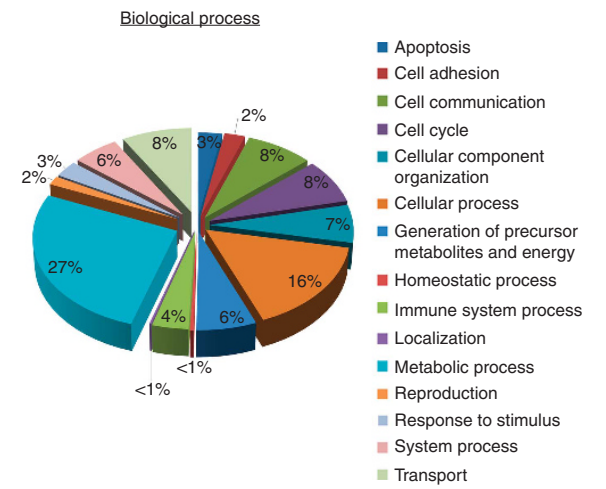

Figure 1. Identification of KSR1-regulated phosphoproteome in breast cancer cells. (A) Experimental schematic outline of SILAC experiment. (B) Scatter plot comparison of phosphosite ratios quantified from control vs KSR1-overexpressed MCF7 cells. (C) Gene ontology (GO) Classification of the KSR1-regulated phosphoproteome in MCF7 cells according to molecular functions, biological processes and cellular compartmentalisation. 
KSR1 abundance. This is by far the first ontology analysis of KSR1regulated phosphoprotein profile in cancer, which will for sure enable extensive study of its functions in signalling pathways other than the canonical Ras-Raf-MAPKs cascades.

Phosphoproteomics analysis reveals a downregulation of phospho-DBC1 by KSR1. Further examination of the identified KSR1-regulated phosphoproteins unveiled that some of these proteins have been previously implicated in the development of breast cancer. For instance, androgen-induced proliferation inhibitor (APRIN), a protein phosphorylated by ATM (ataxia telangiectasiamutated) and ATR (ataxia telangiectasia and Rad3-related) kinases, was described as a BRCA2-interacting protein required for genome integrity and a predictor of outcome in response to chemotherapy in breast cancer (Matsuoka et al, 2007; Brough et al, 2012). Rho GTPase-activating protein 35 (ARHGAP35) was shown to be phosphorylated by breast tumour kinase (Brk) leading to RhoA inactivation, Ras activation and promotion of breast cancer growth and migration (Shen et al, 2008). Phosphorylation of Hsp27 was correlated with HER-2/neu status and lymph node positivity in breast cancer, and phosphorylated Hsp27 was also linked to invasiveness and drug resistance (Zhang et al, 2007; Fujita et al, 2011). Histone deacetylase $1 / \operatorname{Sin} 3 \mathrm{~A}$ complex phosphorylation has been linked to $\mathrm{ER} \alpha$ expression and hormonal therapy resistance in breast cancer cells (De Amicis et al, 2011).

Interestingly, among the most significantly KSR1-regulated phosphorylated proteins, was DBC1, whose involvement in the regulation of p53 activity via SIRT1 has been described previously (Kim et al, 2008; Zhao et al, 2008). Taking into consideration the importance of p53 in breast cancer, we then assessed the connection between KSR1 and p53 activity in vitro.

KSR1 regulates the transcriptional activity of p53. We first examined the effects of KSR1 on p53 transcriptional activity by performing luciferase reporter assays using various p53-dependent gene promoter constructs (including p53-R2, p53-AIP1, p53IGFBP3 and p53-CYCLIN G1). MCF7 cells were co-transfected with either pCMV6 or pCMV6-KSR1 plasmids and each individual p53-dependent promoter construct, in the presence or absence of etoposide, which can induce p53 activity. Interestingly, the luciferase activity of all four different p53-regulated genes was significantly repressed after overexpression of KSR1, suggesting an oppressive role of KSR1 on p53 activation (basal levels and after etoposide treatment; Figure 2A). As expected, silencing of KSR1 resulted in a marked increase in the activity of p53-dependent promoter genes (Figure 2B). These results suggest a potential involvement of KSR1 in p53 transcriptional activity regulation.

KSR1 does not affect the mRNA, protein, subcellular localisation and neddylation levels of p53. In order to investigate the potential mechanism resulting in the downregulation of p53 transcriptional activity, we initially evaluated the p53 gene and protein expression after KSR1 overexpression. RT-qPCR and western blotting analyses did not reveal any changes in the p53 mRNA and protein levels, respectively, after KSR1 transient overexpression in MCF7 cells (Figure 3A). Similarly, no change in p53 protein levels was observed in MCF7 stably overexpressing KSR1 cells comparing with MCF7 parental cells (Figure 3A). As the nuclear localisation of p53 is essential for its activity, we examined whether KSR1 could affect p53 compartmentalisation. Immunofluorescence and subcellular protein fractionation did not reveal any cytoplasmic translocation of p53 upon KSR1 transient overexpression (Figure 3B). Moreover, it has been recently reported that neddylation of p53 can inhibit its transcriptional activity (Xirodimas et al, 2004; Abida et al, 2007). However, our neddylation assay did not show any significant changes following KSR1 overexpression ruling out neddylation as the reason for the observed p53 decreased activity (Figure 3C).

KSR1 decreases p53 acetylation by reducing phosphorylation of DBC1 resulting in impaired DBC1-SIRT1 interaction. Posttranslational modifications of p53 such as phosphorylation and acetylation are essential for p53 activity in response to genotoxic stress (Brooks and Gu, 2003; Tang et al, 2008). To examine effects of KSR1 on phospho-p53 and acetylated-p53, cells were transfected with pCMV6 or pCMV6-KSR1 plasmids in the presence of etoposide or doxorubicin. Although the phosphorylation levels of p53 (Ser15) did not change, interestingly the acetylated-p53 was reduced after KSR1 overexpression upon either etoposide (Figure 4A) or doxorubicin (Supplementary Figure 1) treatment. Similar results were confirmed in several other breast cancer cell lines (including ZR75-1 and SKBR3) and one colon cancer cell line HCT116 (Supplementary Figure 1).

It is already known that SIRT1 functions as an NAD-dependent p53 deacetylase that affects p53 activation (Vaziri et al, 2001; Tang et al, 2008). In addition, DBC1 can interact with and negatively regulate SIRT1 resulting in increased p53 acetylation (Kim et al, 2008; Zhao et al, 2008). Taking this potential link into
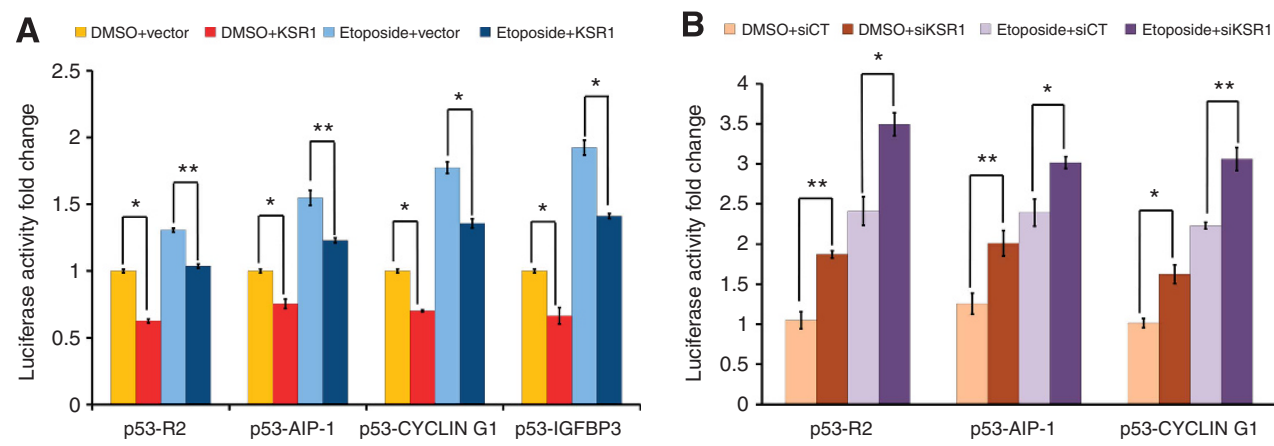

Figure 2. Effects of KSR1 on p53 transcriptional activity in the presence or absence of etoposide by luciferase assays. (A) MCF7 cells were transiently co-transfected with either pCMV6 (vector) or PCMV6-KSR1 plasmids in the presence of four individual p53-dependent promoter constructs expressing firefly luciferase genes (p53-R2, p53-AIP1, p53-CYCLIN G1 and p53-IGFBP3) following dimethylsulphoxide (DMSO) or etoposide $(40 \mu \mathrm{m})$ treatment for $3 \mathrm{~h}$. (B) MCF7 cells were transfected with control siRNA (siCT) or siKSR1 for $48 \mathrm{~h}$, followed by transfection of three p53-dependent promoter constructs expressing firefly luciferase genes (p53-R2, p53-AIP1 and p53-CYCLIN G1) for additional 24h. DMSO or etoposide $(40 \mu \mathrm{M})$ were subsequently added as described above. Firefly luciferase activity was measured (renilla luciferase activity was used to normalise transfection efficiency). The normalised luciferase activity of empty vector is set as 1 . Results shown are the average of at least three independent experiments and error bars represent s.d. Student's $t$-test was performed using SPSS 16.0 statistical software (SPSS Inc.). ( $P<0.05$, $\star \star P<0.01)$. 


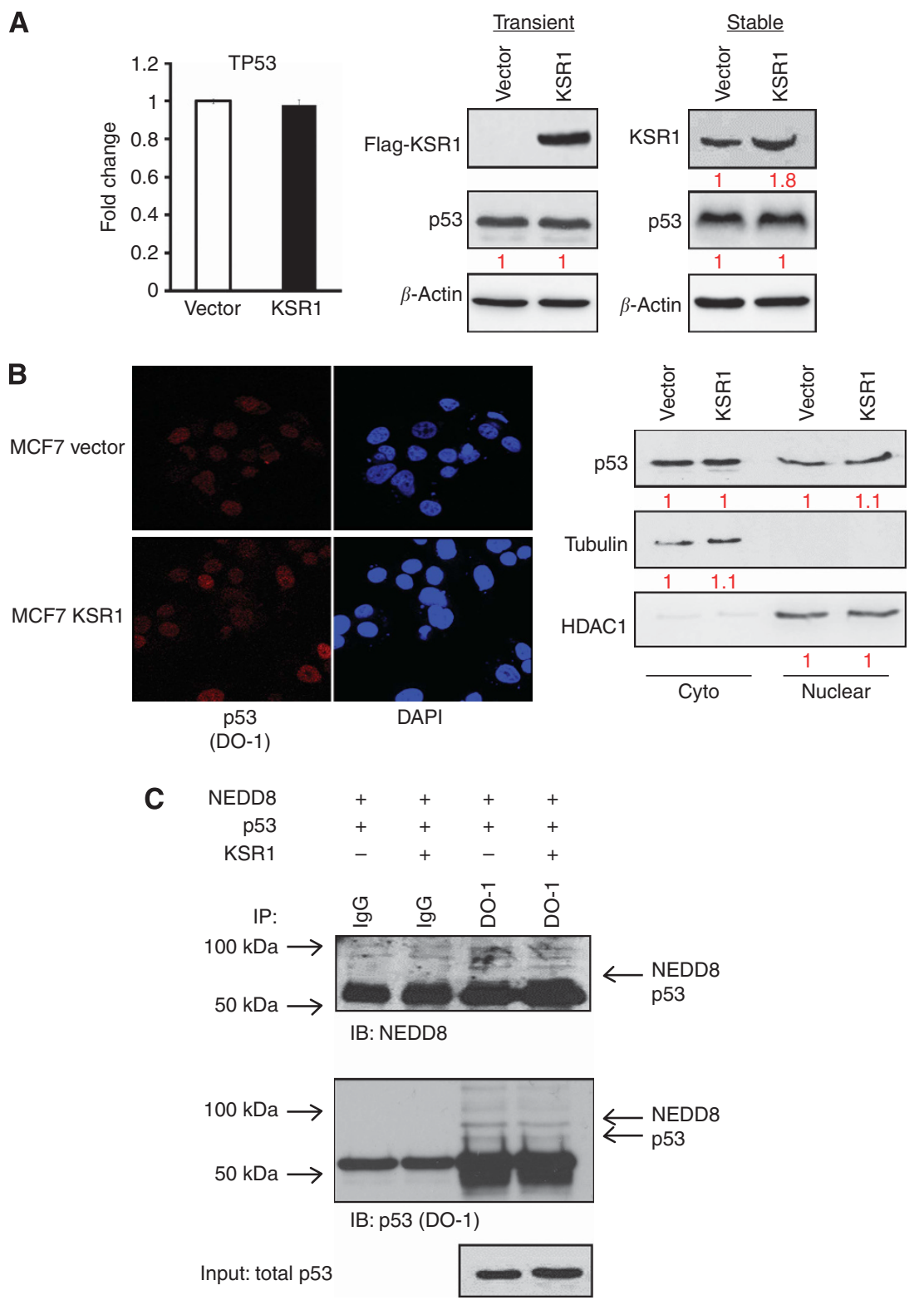

Figure 3. Effects of KSR1 on p53 mRNA, total protein and neddylation levels and on p53 subcellular localisation. (A) Effects on p53 mRNA and total protein levels after KSR1 overexpression. MCF7 cells were transiently transfected with pCMV6 or pCMV6-KSR1 plasmids for $24 \mathrm{~h}$.

Subsequently, relative mRNA levels of TP53 and p53 total protein were measured by RT-qPCR and western blotting, respectively. Gene expression level from cells transfected with pCMV6 was set as 1. Results shown are the average of at least three independent experiments. Similarly, in MCF7 stably overexpressing KSR1 cells, p53 total protein was evaluated by western blot. Blots shown are representatives of at least three independent experiments. (B) Immunofluorescence staining of p53 cells after 24-h transfection with either pCMV6 or pCMV6-KSR1 plasmids in MCF7. p53 was detected with an anti-p53 antibody while the nucleus was stained with 4,6-diamidino-2-phenylindole (DAPI). Representative pictures of three independent experiments are shown. Subcellular fractionation assays were performed after 24-h transfection with either pCMV6 or pCMV6-KSR1 plasmids in MCF7. Tubulin and histone deacetylase 1 (HDAC1) expression served as positive normalising control for cytoplasmic and nuclear proteins respectively. Blots shown are representatives of at least three independent experiments. (C) Neddylation assay on p53 after KSR1 overexpression. MCF7 cells were co-transfected with HA-NEDD8 and pCMV6 or pCMV6-KSR1 plasmids as indicated. p53 was immunoprecipitated using a p53-specific antibody (DO-1) and the neddylated-p53 was detected by immunoblotting using anti-NEDD8 and antip53-specific antibodies. Blots shown are representatives of at least three independent experiments. Abbreviations: IgG = immunoglobulin G; $\mathrm{IP}=$ immunoprecipitation.

consideration, we decided to investigate the effects of KSR1 on these two proteins.

Total SIRT1 levels were not affected by KSR1 upregulation (Figure 4A). Based on the SILAC data regarding the effects of KSR1 on the phosphorylation of $\mathrm{DBC} 1$ and the association between DBC1 and SIRT1, we performed western blotting of phospho-DBC1 (Thr454) revealing a decrease in the phosphorylation levels in basal and etoposide-induced conditions
(Figure 4A). Consistently, KSR1 silencing rescued the abolished acetylation of p53 after etoposide treatment (Figure 4B). Moreover, knock-down of KSR1 did not alter the total proteins of SIRT1 and DBC1, but increased DBC1 phosphorylation at Thr454 (Figure 4B). These data further validate our SILAC results and allow KSR1 to integrate into a known network of p53 acetylation regulation involving phospho-DBC1 and SIRT1. 
A

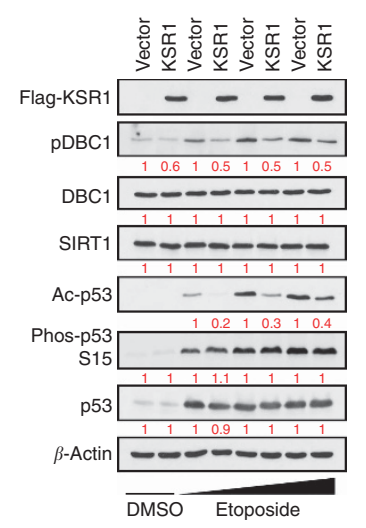

B

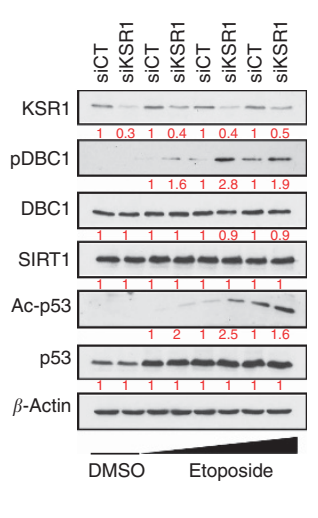

F (i)

IP: $\frac{\text { Input }}{\operatorname{lgG}} \stackrel{\mathrm{DBC} 1}{\square} \leftarrow$ SIRT1

IB: DBC1

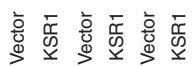

IB: DBC1

IP:

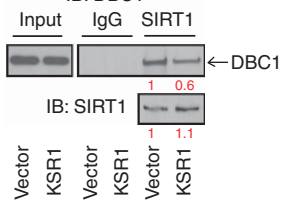

KSR1

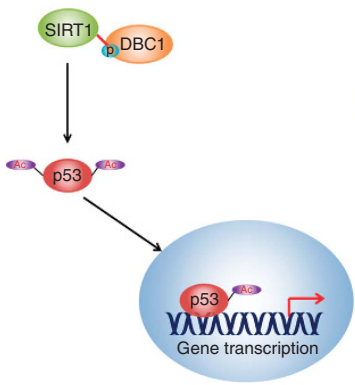

C

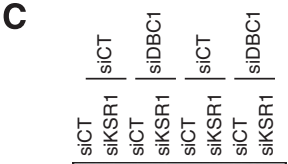

$\mathrm{DBC} 1$

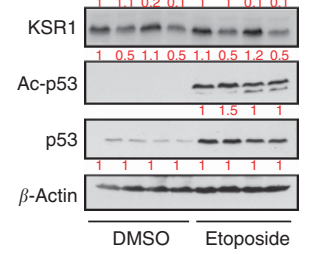

D

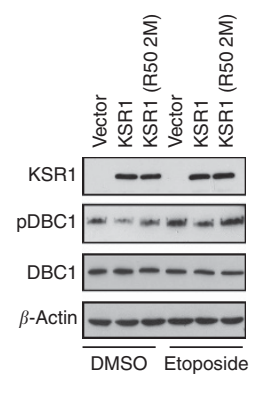

(ii)

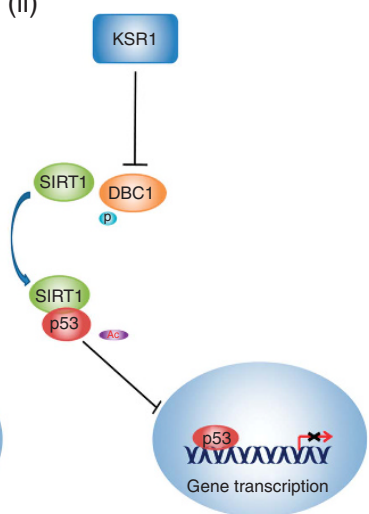

Figure 4. Mechanisms of KSR1-regulated p53 transcriptional activity. (A) Effects on p53 acetylation and phosphorylation of DBC1 after KSR1 overexpression followed by etoposide treatment. MCF7 cells were transiently transfected with pCMV6 (vector) or pCMV6-KSR1 plasmids for $24 \mathrm{~h}$. Subsequently, cells were treated with various concentrations of etoposide (20,40,80 $\mu \mathrm{m}, 3 \mathrm{~h})$. p53 acetylation and DBC1 phosphorylation at Thr454 were assessed by immunoblotting with specific antibodies as indicated. (B) Effects on p53 acetylation and phosphorylation of DBC1 after KSR1 silencing followed by a titration of etoposide treatment. MCF7 cells were transfected with control siRNA (siCT) or siKSR1 for $72 \mathrm{~h}$ followed by etoposide treatment $(20,40,80 \mu \mathrm{M}, 3 \mathrm{~h})$. p53 acetylation and DBC1 phosphorylation at Thr454 were assessed by immunoblotting with specific antibodies as indicated. (C) Effect of KSR1 on p53 acetylation is through DBC1. MCF7 cells were transfected with control siRNA (siCT) or siKSR1 in concordance with siCT or siDBC1 for $72 \mathrm{~h}$ followed by etoposide treatment $(40 \mu \mathrm{m}, 3 \mathrm{~h})$. Acetylated p53, DBC1 and KSR1 protein levels were assessed by immunoblotting with specific antibodies as indicated. (D) Effect of KSR1 on DBC1 phosphorylation is dependent on its intact kinase domain. MCF7 cells were transiently transfected with vector, wild-type KSR1 or mutant KSR1 (R502M) plasmids for $24 \mathrm{~h}$ followed by etoposide treatment $(40 \mu \mathrm{m}, 3 \mathrm{~h})$. DBC1 phosphorylation was measured by immunoblotting with specific antibody. (E) Interaction of DBC1 and SIRT1 after KSR1 overexpression with etoposide treatment by immunoprecipitation (IP). MCF7 cells were transiently transfected with pCMV6 or pCMV6-KSR1 plasmids for $24 \mathrm{~h}$. Subsequently, cells were treated with etoposide $(40 \mu \mathrm{m}, 3 \mathrm{~h})$. The interactions between SIRT1 and DBC1 were detected by IP of SIRT1 or DBC1 followed by immunoblotting with DBC1 and SIRT1 antibodies respectively. Blots shown are representatives of at least three independent experiments. Quantification of blots was analysed by ImageJ software (NIH, Bethesda, MD, USA). (F) Schematic model illustrating the role of KSR1 on p53 transcriptional activity in breast cancer cells with (i) basal or (ii) up-regulated levels of KSR1. Abbreviation: IgG= immunoglobulin G.

To further demonstrate that KSR1 regulates p53 acetylation through DBC1, we studied the effects of KSR1 on p53 acetylation after DBC1 knockdown upon etoposide treatment in MCF7 cells. As shown in Figure 4C, western blotting experiments revealed that acetylated p53 was consistently increased after silencing of KSR1, whereas no change in p53 acetylation was observed after knocking down both KSR1 and DBC1 upon etoposide treatment. This suggests that KSR1 regulates p53 acetylation through $\mathrm{DBCl}$, as depleting DBC1 undermines the effect of KSR1 on acetylated p53.

Moreover, to examine whether the effect of KSR1 on DBC1 depends on its catalytic activity, we generated a KSR1 mutant (KSR1/R502M) that encompasses a key amino-acid mutation (arginine to methionine) within its kinase domain resulting in impaired catalytic activity (Laurent et al, 2004). Consistently, wildtype KSR1 decreased pDBC1, whereas mutant KSR1/R502M sustained $\mathrm{pDBC} 1$ levels in comparison with the control
(Figure 4D). This result suggests that an intact catalytic domain of KSR1 is essential for regulating DBC1 phosphorylation.

Phosphorylation of DBC1, induced by genotoxic stress, creates binding sites and enhances the interaction between SIRT1 and DBC1 (Yuan et al, 2012; Zannini et al, 2012), which subsequently undermines the deacetylase activity of SIRT1 on p53. For this reason, we tested whether KSR1 affects the stress-induced SIRT1$\mathrm{DBC} 1$ interaction. As shown in Figure 4E, upon etoposide treatment, the interaction between SIRT1 and DBC1, determined by co-immunoprecipitation (IP), was undermined after KSR1 overexpression in MCF7, which allows SIRT1 to interact more with p53 resulting in declined p53 acetylation.

KSR1 is important for breast cancer proliferation and its expression is altered in breast cancer. To study whether KSR1 affects breast cancer growth in vitro, cell proliferation assay was 
performed in four different breast cancer cell lines. As shown in Figure 5, knockdown of KSR1 significantly reduced breast cancer cell growth in MCF7, ZR75-1, SKBR3 and MDA231 after six days, showing an important role of KSR1 in breast cancer cell proliferation. This is also supported by further examination of KSR1 expression in breast tumour tissues. Oncomine analysis (Rhodes et al, 2004) was conducted to study the levels of KSR1 in normal breast and cancer tissues from TCGA Breast database. Indeed, KSR1 abundance is varied between normal breast and various breast carcinomas, including invasive ductal breast carcinoma and invasive lobular breast carcinoma (Figure 6). Specifically, KSR1 expression is significantly upregulated in invasive ductal breast carcinoma, lobular breast carcinoma and invasive breast carcinoma.

\section{DISCUSSION}

KSR1 was originally identified as a novel protein kinase evolutionarily conserved in Drosophila and Caenorhabditis elegans functioning between Ras and Raf in the Ras-Raf-MAPKs signalling pathway (Kornfeld et al, 1995; Therrien et al, 1995). However, mammalian KSR1 has been extensively referred as a pseudokinase, because of the mutation in the lysine residue in the catalytic domain, which is essential for its kinase activity (Zhang et al, 2012). Later, the role of KSR1 as a scaffolding protein was discovered. Murine KSR1 was first reported to cooperate with activated Ras to facilitate MAPK kinases activation thus stimulating Xenopus oocyte maturation and cellular transformation (Therrien et al, 1996). At the same time, the idea of KSR1 as an active kinase was described from the finding that TNF- $\alpha$ and ceramide were shown to significantly increase KSR1 autophosphorylation and its capacity to phosphorylate and activate Raf-1 (Zhang et al, 1997). Therefore, emerging evidence suggests dual function of KSR1 as an active kinase as well as a scaffold protein
(Rajakulendran et al, 2009; Hu et al, 2011). Moreover, KSR1 has been previously shown as an oncogene in Ras-dependent cancers, such as pancreatic and lung carcinomas (Xing et al, 2003; Kortum and Lewis, 2004). However, its biological functions and modulated signalling pathways in breast cancer have remained undefined.

In this study, we performed a global comparative proteomic analysis and for the first time reveal the complex KSR1-regulated phosphoproteome in MCF7 breast cancer cells. Various new KSR1-regulated proteins and signalling networks that KSR1 is involved in were identified. Notably, our phosphoproteomic analysis showed that the majority (233 out of 379) of the identified phospho-sites were actually reduced after KSR1 overexpression in comparison with a small number of increased phosphorylations ( 3 out of the 379), suggesting its primary role as a scaffold protein not a kinase at least in this context. Interestingly, none of the previously described proteins including MEK and ERK in the canonical Ras-Raf-MAPKs pathways have been identified in our study. The possible explanation could be that the Ras mutations are very rare in breast cancer (Adjei, 2001). Indeed, our recent work has also shown no significant alteration in phosphorylation of the main components in the Ras-Raf-MAPKs cascades upon overexpressing or silencing KSR1 (unpublished work). Moreover, the KSR1-regulated phosphoproteins identified in this study illustrated a much more extensive view of KSR1 involved biological functions, including cell cycle, metabolism and apoptosis. Notably, some phosphoproteins have been shown to have vital roles in multiple aspects of breast cancer. For example, APRIN was reported as a BRCA2interacting protein essential for genome integrity and associated with chemotherapy response in breast cancer (Matsuoka et al, 2007; Brough et al, 2012), whereas ARHGAP35 was shown to be important in breast cancer growth and migration (Shen et al, 2008). Phospho-Hsp27 was related to invasiveness and drug resistance in breast cancer (Zhang et al, 2007; Fujita et al, 2011). Most importantly, the KSR1-regulated phosphoproteomic
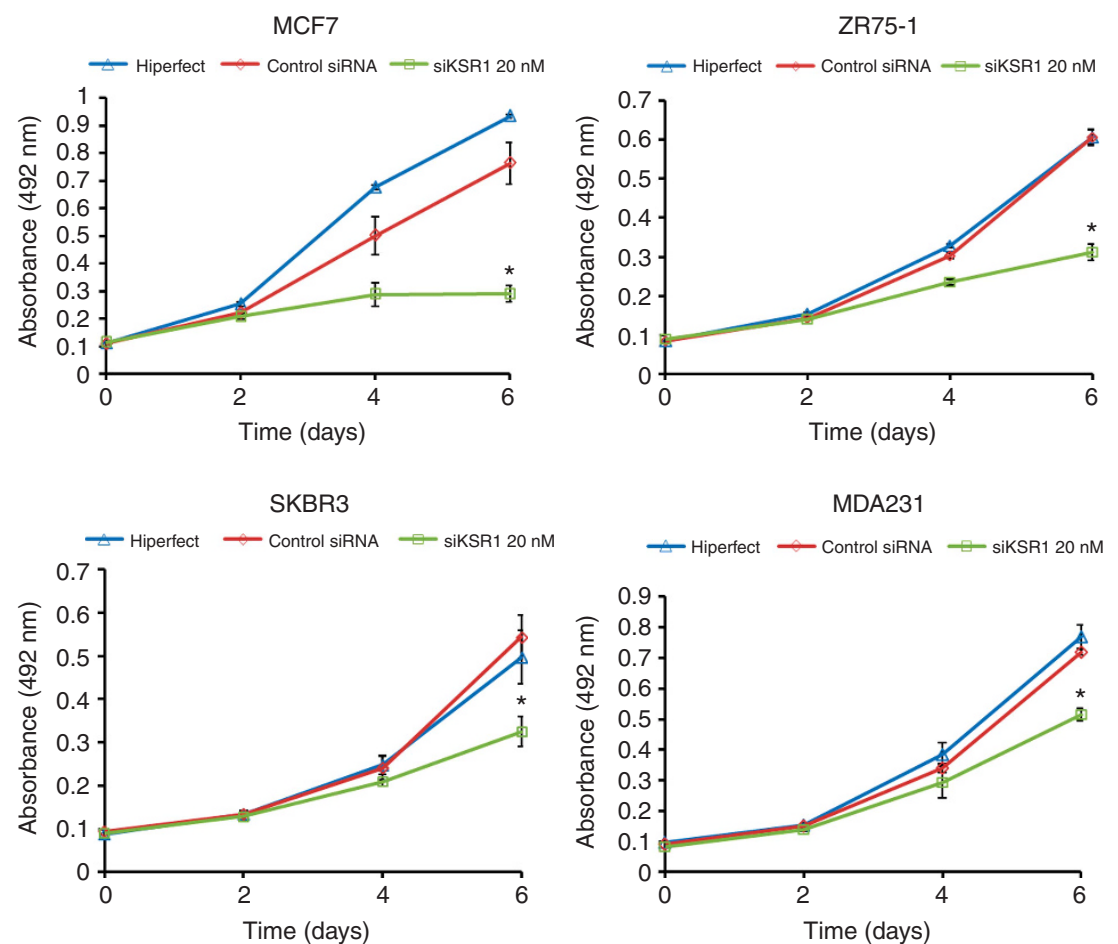

Figure 5. Effects of KSR1 silencing on breast cancer cell proliferation in vitro. SRB assays of MCF7, ZR75-1, SKBR3 and MDA231 cells after transfection with $20 \mathrm{~nm}$ of either siKSR1 or 'non-targeting' siRNA (control siRNA) or vehicle (Hiperfect) for 6 days. Error bars represent s.d. of three experiements each in quintuplicates ( ${ }^{*} P<0.05$, compared with control siRNA at day 6; Student's $t$-test). 

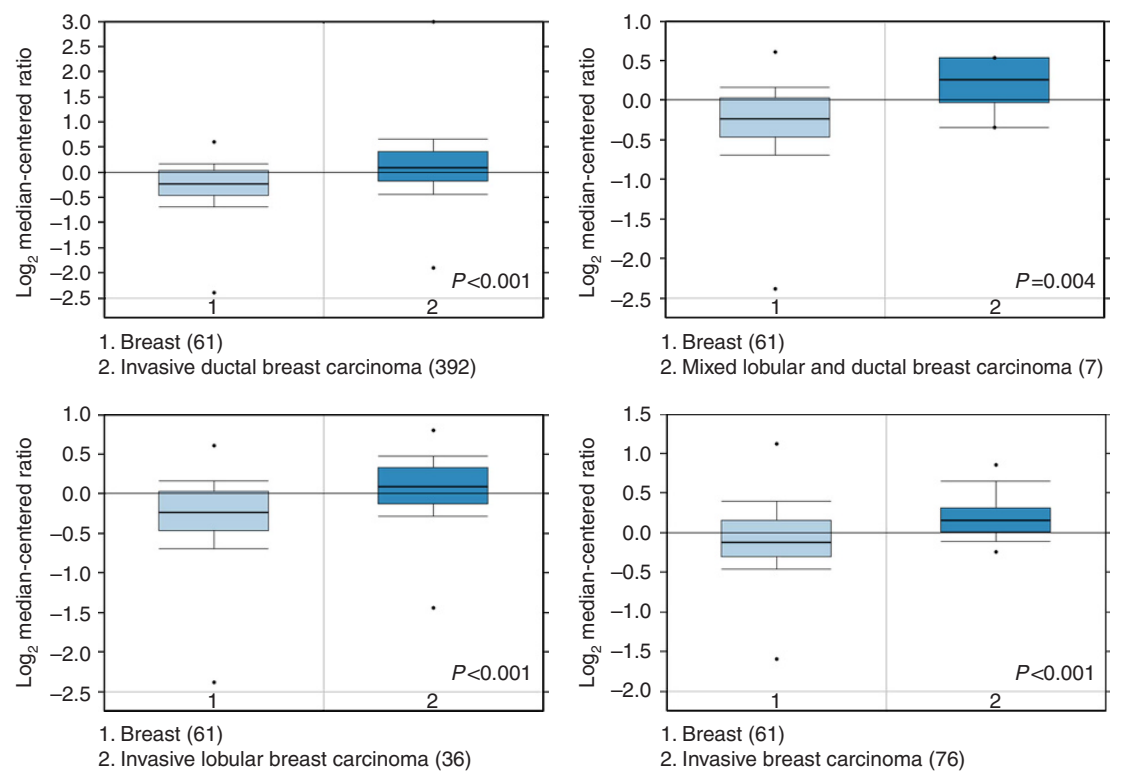

Figure 6. KSR1 expression is altered in breast cancer tissues. Oncomine analysis was performed to examine KSR1 expression in breast normal and cancer tissues using online TCGA microarray data (www.oncomine.org).

analysis has identified phospho-DBC1 as a potential connection to its modulation of p53 transcriptional activity here.

Deleted in breast cancer-1 was previously reported to be involved in regulating p53 acetylation (Kim et al, 2008; Zhao et al, 2008), which is indispensable for p53 transcriptional activity (Tang et al, 2008). Therefore, our work further investigated the effects of KSR1 on p53 transcriptional activity, as well as the potential mechanism on KSR1-mediated regulation of p53 transcriptional activity, including p53 acetylation. Luciferase assays using different p53-dependent gene promoter constructs showed that KSR1 overexpression suppressed p53 transcriptional activity in the absence or presence of etoposide, whereas the opposite results were observed after silencing of KSR1, indicating a regulatory role of KSR1 in p53 activity. Mechanistic study demonstrated that overexpression of KSR1 had no effect on p53 mRNA and total protein levels, as well as subcellular localisation, phosphorylation and neddylation, which are all potentially involved in p53 activity modulation.

As expected, KSR1 overexpression reduced the acetylation of p53, whereas silencing of KSR1 rescued the decreased p53 acetylation in the absence or presence of etoposide, suggesting that KSR1 is involved in regulating p53 acetylation. It has already been shown that $\mathrm{DBC} 1$ can interact with and negatively regulate SIRT1, a NAD-dependent p53 deacetylase, resulting in increased p53 acetylation (Kim et al, 2008; Zhao et al, 2008). Moreover, phospho-DBC1, induced by genotoxic stress, is competent to create binding sites and enhance the interaction between SIRT1 and DBC1, which subsequently undermines deacetylase activity of SIRT1 on p53 (Yuan et al, 2012; Zannini et al, 2012). Consistently, we demonstrated that KSR1 overexpression decreases the phospho-DBC1 levels, whereas KSR1 silencing increases DBC1 phosphorylation. This provides us an explanation to the observed effects of KSR1 on p53 acetylation and activity. Similar results were obtained after overexpression or silencing KSR1 in different breast cancer cell lines including ZR75-1 and SKBR3. Further co-IP experiments proved that overexpression of KSR1 attenuates the interaction of SIRT1-DBC1, which therefore allows SIRT1 to interact more with p53 resulting in decreased p53 acetylation. Our data herein are consistent with previous reports and in combination with our SILAC analyses enable KSR1 to integrate into a known network together with DBC1 and SIRT1 on regulation of p53 acetylation.
However, more evidence is still needed to elucidate the mechanism of KSR1 on modulating DBC1 phosphorylation. The work from Zannini et al (2012) demonstrated that ATM/ATR can directly phosphorylate $\mathrm{DBC} 1$ on Thr454 upon DNA damage. Phosphorylated DBC1 bound to and inhibited SIRT1, leading to the separation of the SIRT1-p53 complex and an increase of p53 acetylation. Moreover, another group indicated that protein kinase A and AMP-activated protein kinase was capable of inducing the dissociation of SIRT1 from its endogenous inhibitor DBC1, resulting in an alteration in downstream effects (Nin et al, 2012). Therefore, whether the effect of KSR1 on DBC1 phosphorylation is through ATM/ATR kinases or alternative pathways requires further investigation.

Collectively, our SILAC analyses of the KSR1-regulated phosphoproteome profile in cancer cells demonstrate its involvement in multiple biological and molecular processes as well as intricate signalling pathways. The identification of novel KSR1regulated proteins will shed light on new KSR1-modulated signalling pathways implicated in breast cancer. In this work, we propose a model (Figure $4 \mathrm{~F}$ ) where in cancer cells with basal levels of KSR1, phosphorylated DBC1 directly interacts with SIRT1 and reduces its deacetylase activity resulting in p53 activation and gene expression initiation. However, in KSR1-transduced cells, KSR1 suppresses DBC1 phosphorylation, which undermines the direct interaction between DBC1 and SIRT1. This in turn allows SIRT to decrease p53 acetylation, which eventually inhibits p53 transcriptional activity. Our study provides a new insight on the role of KSR1 in p53 regulation and unveils an interesting mechanism for its function in breast cancer development.

\section{ACKNOWLEDGEMENTS}

We thank Arnhild Grothey, Ylenia Lombardo and Chun Fui Lai for their technical assistance. This work was supported by the National Institute for Health Research, the Pink Ribbon Foundation, Action Against Cancer, China Scholarship Council, Breast Cancer Campaign and Cancer Research UK. We thank Richard and Evelina Girling and the 'kinase group' for their support. This work is dedicated to Padroic Fallon, his family and friends. 


\section{CONFLICT OF INTEREST}

The authors declare no conflict of interest.

\section{REFERENCES}

Abida WM, Nikolaev A, Zhao W, Zhang W, Gu W (2007) FBXO11 promotes the Neddylation of p53 and inhibits its transcriptional activity. J Biol Chem 282: $1797-1804$.

Adjei AA (2001) Blocking oncogenic Ras signaling for cancer therapy. J Natl Cancer Inst 93: 1062-1074.

Brooks CL, Gu W (2003) Ubiquitination, phosphorylation and acetylation: the molecular basis for p53 regulation. Curr Opin Cell Biol 15: 164-171.

Brough R, Bajrami I, Vatcheva R, Natrajan R, Reis-Filho JS, Lord CJ, Ashworth A (2012) APRIN is a cell cycle specific BRCA2-interacting protein required for genome integrity and a predictor of outcome after chemotherapy in breast cancer. EMBO J 31: 1160-1176.

Cox J, Mann M (2008) MaxQuant enables high peptide identification rates, individualized p.p.b.-range mass accuracies and proteome-wide protein quantification. Nat Biotechnol 26: 1367-1372.

Cox J, Neuhauser N, Michalski A, Scheltema RA, Olsen JV, Mann M (2011) Andromeda: a peptide search engine integrated into the MaxQuant environment. J Proteome Res 10: 1794-1805.

De Amicis F, Giordano F, Vivacqua A, Pellegrino M, Panno ML, Tramontano D, Fuqua SA, Ando S (2011) Resveratrol, through NF-Y/p53/Sin3/HDAC1 complex phosphorylation, inhibits estrogen receptor alpha gene expression via p38MAPK/CK2 signaling in human breast cancer cells. FASEB J 25: 3695-3707.

Fujita R, Ounzain S, Wang AC, Heads RJ, Budhram-Mahadeo VS (2011) Hsp-27 induction requires POU4F2/Brn-3b TF in doxorubicin-treated breast cancer cells, whereas phosphorylation alters its cellular localisation following drug treatment. Cell Stress Chaperones 16: 427-439.

Giamas G, Filipovic A, Jacob J, Messier W, Zhang H, Yang D, Zhang W, Shifa BA, Photiou A, Tralau-Stewart C, Castellano L, Green AR, Coombes RC, Ellis IO, Ali S, Lenz HJ, Stebbing J (2011) Kinome screening for regulators of the estrogen receptor identifies LMTK3 as a new therapeutic target in breast cancer. Nat Med 17: 715-719.

Goettel JA, Liang D, Hilliard VC, Edelblum KL, Broadus MR, Gould KL, Hanks SK, Polk DB (2011) KSR1 is a functional protein kinase capable of serine autophosphorylation and direct phosphorylation of MEK1. Exp Cell Res 317: 452-463.

Hu J, Yu H, Kornev AP, Zhao J, Filbert EL, Taylor SS, Shaw AS (2011) Mutation that blocks ATP binding creates a pseudokinase stabilizing the scaffolding function of kinase suppressor of Ras, CRAF and BRAF. Proc Natl Acad Sci USA 108: 6067-6072.

Kim JE, Chen J, Lou Z (2008) DBC1 is a negative regulator of SIRT1. Nature 451: 583-586.

Kolesnick R, Xing HR (2004) Inflammatory bowel disease reveals the kinase activity of KSR1. J Clin Invest 114: 1233-1237.

Kornfeld K, Hom DB, Horvitz HR (1995) The ksr-1 gene encodes a novel protein kinase involved in Ras-mediated signaling in C. elegans. Cell 83: 903-913.

Kortum RL, Lewis RE (2004) The molecular scaffold KSR1 regulates the proliferative and oncogenic potential of cells. Mol Cell Biol 24: 4407-4416.

Laurent MN, Ramirez DM, Alberola-Ila J (2004) Kinase suppressor of Ras couples Ras to the ERK cascade during T cell development. J Immunol 173: 986-992.

Levine AJ (1997) p53, the cellular gatekeeper for growth and division. Cell 88: 323-331.

Lozano J, Xing R, Cai Z, Jensen HL, Trempus C, Mark W, Cannon R, Kolesnick R (2003) Deficiency of kinase suppressor of Ras1 prevents oncogenic ras signaling in mice. Cancer Res 63: 4232-4238.

Matsuoka S, Ballif BA, Smogorzewska A, McDonald 3rd ER, Hurov KE, Luo J, Bakalarski CE, Zhao Z, Solimini N, Lerenthal Y, Shiloh Y, Gygi SP, Elledge SJ (2007) ATM and ATR substrate analysis reveals extensive protein networks responsive to DNA damage. Science 316: 1160-1166.
Mi H, Muruganujan A, Thomas PD (2013) PANTHER in 2013: modeling the evolution of gene function, and other gene attributes, in the context of phylogenetic trees. Nucleic Acids Res 41: D377-D386.

Nin V, Escande C, Chini CC, Giri S, Camacho-Pereira J, Matalonga J, Lou Z, Chini EN (2012) Role of deleted in breast cancer 1 (DBC1) protein in SIRT1 deacetylase activation induced by protein kinase A and AMPactivated protein kinase. J Biol Chem 287: 23489-23501.

Rajakulendran T, Sahmi M, Lefrancois M, Sicheri F, Therrien M (2009) A dimerization-dependent mechanism drives RAF catalytic activation. Nature 461: 542-545.

Rhodes DR, Yu J, Shanker K, Deshpande N, Varambally R, Ghosh D, Barrette T, Pandey A, Chinnaiyan AM (2004) ONCOMINE: a cancer microarray database and integrated data-mining platform. Neoplasia 6: 1-6.

Shen CH, Chen HY, Lin MS, Li FY, Chang CC, Kuo ML, Settleman J, Chen RH (2008) Breast tumor kinase phosphorylates p190RhoGAP to regulate rho and ras and promote breast carcinoma growth, migration, and invasion. Cancer Res 68: 7779-7787.

Tang Y, Zhao W, Chen Y, Zhao Y, Gu W (2008) Acetylation is indispensable for p53 activation. Cell 133: 612-626.

Therrien M, Chang HC, Solomon NM, Karim FD, Wassarman DA, Rubin GM (1995) KSR, a novel protein kinase required for RAS signal transduction. Cell 83: 879-888.

Therrien M, Michaud NR, Rubin GM, Morrison DK (1996) KSR modulates signal propagation within the MAPK cascade. Genes Dev 10: 2684-2695.

Thingholm TE, Jorgensen TJ, Jensen ON, Larsen MR (2006) Highly selective enrichment of phosphorylated peptides using titanium dioxide. Nat Protoc 1: 1929-1935.

Vaziri H, Dessain SK, Ng Eaton E, Imai SI, Frye RA, Pandita TK, Guarente L, Weinberg RA (2001) hSIR2(SIRT1) functions as an NAD-dependent p53 deacetylase. Cell 107: 149-159.

Vikhanskaya F, Lee MK, Mazzoletti M, Broggini M, Sabapathy K (2007) Cancer-derived p53 mutants suppress p53-target gene expressionpotential mechanism for gain of function of mutant p53. Nucleic Acids Res 35: 2093-2104.

Vogelstein B, Lane D, Levine AJ (2000) Surfing the p53 network. Nature 408: 307-310.

Wisniewski JR, Zougman A, Nagaraj N, Mann M (2009) Universal sample preparation method for proteome analysis. Nat Methods 6: 359-362.

Xing HR, Cordon-Cardo C, Deng X, Tong W, Campodonico L, Fuks Z, Kolesnick R (2003) Pharmacologic inactivation of kinase suppressor of ras-1 abrogates Ras-mediated pancreatic cancer. Nat Med $\mathbf{9}$ : $1266-1268$.

Xirodimas DP, Saville MK, Bourdon JC, Hay RT, Lane DP (2004) Mdm2mediated NEDD8 conjugation of p53 inhibits its transcriptional activity. Cell 118: 83-97.

Yuan J, Luo K, Liu T, Lou Z (2012) Regulation of SIRT1 activity by genotoxic stress. Genes Dev 26: 791-796.

Zafrullah M, Yin X, Haimovitz-Friedman A, Fuks Z, Kolesnick R (2009) Kinase suppressor of Ras transphosphorylates c-Raf-1. Biochem Biophys Res Commun 390: 434-440.

Zannini L, Buscemi G, Kim JE, Fontanella E, Delia D (2012) DBC1 phosphorylation by ATM/ATR inhibits SIRT1 deacetylase in response to DNA damage. J Mol Cell Biol 4: 294-303.

Zhang D, Wong LL, Koay ES (2007) Phosphorylation of Ser78 of Hsp27 correlated with HER-2/neu status and lymph node positivity in breast cancer. Mol Cancer 6: 52 .

Zhang H, Photiou A, Grothey A, Stebbing J, Giamas G (2012) The role of pseudokinases in cancer. Cell Signal 24: 1173-1184.

Zhang Y, Yao B, Delikat S, Bayoumy S, Lin XH, Basu S, McGinley M, Chan-Hui PY, Lichenstein H, Kolesnick R (1997) Kinase suppressor of Ras is ceramide-activated protein kinase. Cell 89: 63-72.

Zhao W, Kruse JP, Tang Y, Jung SY, Qin J, Gu W (2008) Negative regulation of the deacetylase SIRT1 by DBC1. Nature 451: 587-590.

This work is published under the standard license to publish agreement. After 12 months the work will become freely available and the license terms will switch to a Creative Commons AttributionNonCommercial-Share Alike 3.0 Unported License.

Supplementary Information accompanies this paper on British Journal of Cancer website (http://www.nature.com/bjc) 